\title{
Indications for the sentinel node: multicentric, size of tumor, prior surgery
}

\author{
L. C. Hanker, A. Rody, E. Ruckhaeberle, M. Kaufmann \\ Department of Gynaecology and Obstetrics, Johann Wolfgang Goethe-University, Frankfurt/Main, Germany
}

\begin{abstract}
Sentinel lymph node biopsy (SLNB) has been adopted as an applicable alternative to the standard axillary lymph node dissection (ALND) level I and II. It makes possible a less extensive axillary surgery in patients with early breast cancer with negative lymph node, who would not benefit from further dissection, in order to prevent unnecessary morbidity. On the other hand, SLNB is not appropriate in every clinical circumstance. In some clinical situations like tumor size T1 and T2, SLNB is, meanwhile, regarded as a standard procedure. In other settings like increased age and body mass index, pregnancy, ductal carcinoma in situ (DCIS), neoadjuvant chemotherapy, advanced disease (T3 and T4), prior surgery and multifocal/ multicentric disease, there is a controversial debate about the importance of SLNB. This article reviews the absolute and relative contraindications of this procedure in respect to the latter three clinical situations.

For the advanced breast cancers T3 and T4, there seems to be an increasing evidence of an acceptable accuracy, although it should be further evaluated in randomized clinical trials (RCT). The indication of SLNB in previously operated patients depends on the type of prior surgery. A diagnostic biopsy does not represent a contraindication, whereas the sentinel node biopsy should not be used after an extensive breast surgery neither in the context of oncologic nor non-oncologic purposes. In the context of multicentric disease, there is growing evidence that SLNB is suitable, but actually it should be restricted to RCT. However, the multifocal disease is only a relative contraindication, that is it could be applied in well-selected patients.
\end{abstract}

Keywords: Axillary dissection; Multicentric disease; Sentinel node

\section{Introduction}

Axillary lymph node dissection (ALND) is a standard procedure for the surgical treatment of early breast cancer. It is not only important for the treatment of this disease, that is reducing the local recurrence rate by removing metastatic lymph nodes, but also for the diagnostic procedure. The degree of the

Correspondence to: L. C. Hanker, MD, Department of Gynaecology and Obstetrics, Johann-Wolfgang-Goethe University, TheodorStern-Kai 7, 60590 Frankfurt/Main, Germany. E-mail: hanker@med.uni-frankurt.de; Tel: +49(0)696301 7024; Fax: +49(0)6963017938

Received: 06/07/07

Accepted: 18/12/07

BCO/611/2007/FO axillary lymph node invasion is important for the accurate staging and choice of the adequate adjuvant therapy.

The procedure of sentinel lymph node biopsy (SLNB) has become a widely accepted alternative to the ALND and helps to identify patients with negative axillary lymph nodes in order to prevent an unnecessary axillary dissection which represents no benefit for these women, but is additionally associated with morbidity from this procedure, that is increased risk of infection, decreased range of motion and lymphedema [1].

SLNB is regarded as a standard surgical procedure by the most experts and panels for women with T1- and T2-tumors, usually less than $3 \mathrm{~cm}$ in 
diameter [2]. In addition, a clinically negative axilla $c(N 0)$ is required, that is there should not be any suspicious palpable axillary lymph nodes. Apart from these clear indications and contraindications, there are some special clinical conditions in which it is not clear whether SLNB represents a relative/ absolute contraindication or not.

Patients with increased age, increased body mass index, pregnancy, ductal carcinoma in situ (DCIS), neoadjuvant chemotherapy, advanced disease (T3 and T4), prior surgery and mulifocal/ multicentric disease delineate special circumstances for which indications and limitations of SLNB are not clearly defined. In this review, the indications for the latter three are further evaluated.

\section{Multicentric/multifocal breast cancer}

The role of SLNB in multicentric and multifocal disease in breast cancer is not yet clearly defined. Multicentricity is described as the presence of more than one cancer spots in the breast, occurring in separate quadrants or at a distance of more than $2-5 \mathrm{~cm}$ from each other [3]. In contrast to that definition, the multifocal disease is referred to the presence of several foci in the same quadrant of the breast.

It occurs in approximately $10-63 \%[3,4]$ of the cases and may result in inaccurate lymph node staging and high false-negative rates after an SLNB $[5,6]$. The most trials addressing SLNB have excluded women with multicentric or multifocal lesions. Therefore, this topic is not clearly evaluated and a recommendation is difficult to give. Most experts and panelists have been considered multicentricity/multifocality as a relative contraindication because of the suspicion that several tumor spots might drain to more than one dominant lymphatic pathway and sentinel lymph node (SLN). However, this point of view begins to change. At the beginning of SLNB era, this biopsy technique was used by injecting either radiolabeled colloid or blue dye peritumorly, that is directly near the tumor lesion $[7,8]$. Recently some trials have evaluated different sites of injection like intradermal $[9,10]$, subdermal [5] or subareolar $[11,12]$. It has been shown that these injection sites were associated even with greater success and a comparable false negative rate to that associated with the standard injection site [13]. These results support the theory that all quadrants of the breast drain into the same lymph node. Assuming the idea that the whole breast drains to the same SLN, one could suggest that SLNB can even be performed in multicentric and multifocal cancer. This theory is supported by several authors [12,14-16]. Additionally, some investigators were able to show that the test performance of SLNB in multicentric and multifocal disease is nearly equivalent to that for unifocal breast cancer [17-19]. Identification rates ranged from $90 \%$ to $97 \%$ [20] and false negative rates from $0 \%$ to $8 \%$ [19]. Knauer et al. [20] reported on a multi-institutional trial in which the SNB-feasibility and accuracy was evaluated in 142 patients with multicentric breast cancer. Compared to patients with unicentric cancer it has been shown a higher rate of SN metastases, whereas there was no difference in sensitivity (96.0\%), negative predictive value (93.3\%) and overall accuracy (97.3\%) [20].

Nevertheless, it should be pointed out that the above-mentioned studies were only small and non-randomized series, so that further evaluations of this procedure have to be done. Therefore, we finally conclude with the German Society of Senology and point out that there is not sufficient evidence to recommend the procedure of SLNB in multicentric disease outside of randomized trials for routine clinical practice [21]. However, the multifocal breast cancer should not represent a contraindication according to the leading professional societies and published trials $[2,21,22]$.

\section{Prior surgery - previous biopsy}

The impact of prior surgery of the breast and the axilla on accurate lymphatic mapping has not been evaluated very well either. On one hand, there are the oncologic biopsy procedures like incisional, excisional or core biopsy, which seem not to affect the success of SLNB according to some limited data [23]. On the other hand, there are the non-oncologic and oncologic procedures like reduction and augmentation mammoplasty or breast reduction that are not sufficiently examined. Neither the breast surgery nor the axillary surgery (i.e. mammoplasty through an axillary incision) is fully evaluated.

Concerning the prior breast surgery with the aim of a diagnostic biopsy, growing data suggest that the success of SLNB is not affected [23-27]. Nevertheless, it has to be emphasized that many previous trials on SLNB have excluded women with excisional biopsy [28] and/or previous axillary surgery [29]. Additionally, some authors reported on a higher failure rate after an excisional biopsy [29-31], whereas others found promising results. Miner et al. [32] were able to clarify that the type of previously performed diagnostic biopsy or the location of the primary lesion did not influence the localization of the SLN. In another study it has been shown that excision done before SLNB did not interfere with the subsequent identification rate of the SLN [33]. It could be shown that neither the excision volume 
nor the time interval between the diagnostic biopsy and the lymph node mapping did play a role in affecting the success of SLNB [23]. So, taking into account the available data, a prior biopsy seems not to influence the successful lymphatic mapping.

The other group of prior breast surgery with nononcologic and oncologic aims has been less examined. The effect on the identification rates of the SLN in women who have undergone a nononcologic breast surgery, for example reduction mammoplasty, is not clearly known. Probably, the most important point for the correct identification of the SLN is the presence of an intact lymphatic pathway between the tumor and the axilla. If the tumor is situated in the upper quadrant, it is likely that, for example a reduction procedure does not affect the lymphatic drainage. Therefore, a sentinel procedure could be done, especially when prior surgery was performed more than 6-12 month previously. In the re-operative oncologic setting, it could be speculated that the intact lymphatic pathway seems to be as essential as in the nononcologic setting for a successful identification of the sentinel node. Up to date, there are no randomized clinical trials (RCT) concerning this special situation.

The impact of the axillary surgery also remains uncertain. In a retrospective trial it was pointed out that SLNB after prior axillary surgery, like ALND or SLNB, is associated with a higher rate of failure, of $25 \%$ [34]. Although there have been only 32 cases in this study that have had an attempted sentinel biopsy after prior axillary surgery, this result indicates a negative effect of repeated SLNB. On the other hand, Intra et al. [35] published data from a single institution trial that show feasibility and efficacy of SLNB in the re-operative setting. The authors demonstrated an identification rate of $87 \%$ in patients with 10 or less axillary lymph nodes previously removed [35]. However, in patients with more than 10 lymph nodes excised, the rate decreased to $44 \%$. The recently published results of Port et al. [36] support these data. The authors were able to show in an unicenter trial that the success of re-operative SLNB was inversely related to the number of nodes removed previously and was more likely to be successful after a previous SLNB than a previous ALND (74\% vs. 38\%) [36].

Taken together, these results suggest that SLNB should not be performed after previous axillary surgery, neither in the oncologic setting nor in the non-oncologic setting. However, in a selected group of patients this procedure could be taken into account, especially after prior SLN procedure. SLNB can be performed in patients who have had a previous excisional biopsy and even in patients after a limited non-oncologic breast surgery [22]. In summery, the application of SLNB after extensive previous breast surgery has a diminished identification rate and should therefore be contraindicated.

\section{Large and locally advanced breast cancer}

Most experts recommend the procedure of SLNB in T1 and T2 tumors [2]. Although there is only one randomized and controlled published trial in which SLNB was compared with ALND for the effects on long-term survival [37], there is a strong evidence for the high quality of test performance of SLNB in patients with early breast cancer, given by the numerous amount of test performance trials [2]. Nevertheless, it should be emphasized that most validation trials included only patients with $T 1$ and T2 tumors. A recently published meta-analysis and three other additional systematic reviews have underlined the advantage and quality of this procedure in this special group of women [2,38-40]. As stated in another representative multicenter setting, the sensitivity of SLNB is about $90 \%$ and the false negative rate is about $10 \%[25,29]$ for early breast cancer. However, for the locally advanced and larger T3 and T4 tumors, there are also a few reports that describe SLNB in these patients. Some authors did reveal that the identification rate and the sensitivity of SLNB in patients with smaller tumors were equivalent to those with breast tumors larger than $4 \mathrm{~cm}$ [41]. Chung et al. [42] reported on an acceptable accuracy rate in patients with larger tumors $(\geqslant 5 \mathrm{~cm})$. In this trial, the SLN status accurately predicted the regional nodal status in $98 \%$ (40 of 41) of cases [42]. Wong et al. [43] published data of an analysis that was performed to determine whether tumor size affects the accuracy of SLNB. The SLN identification rate, false negative rate and overall accuracy of SLNB were not significantly different among tumor stages T1, T2 and T3 [43]. Another study did reveal similar results. The SLN was identified in $99 \%$ of cases. SLNB false-negative rate was $2 \%$ [44]. Although these data [41-44] seem to support the statement that tumor size does not harm the sensitivity, we conclude with the recommendation that SLNB should not be used routinely for this group of patients because of the small amount of RCT. In a selected group of patients with advanced breast cancer, SLNB has also been used after neoadjuvant chemotherapy [45]. It could be shown an identification rate between $85 \%$ and $94 \%$ [46-48] and a false negative rate of $12 \%$ [46]. These data seem to indicate a potent role for SLNB in this setting. Nevertheless, there have to be more trials including large numbers of patients to clarify this indication of SLNB [49]. In the meantime, 
SLNB after neoadjuvant chemotherapy should be restricted to the hands of experts with adequate experience [50].

\section{Conclusion}

SLNB is not appropriate for every patient. In some conditions, as for example multicentric disease, it should not be used outside clinical trials. However, the multifocal disease is only a relative contraindication, that is it could be applied in well-selected patients.

The indication of SLNB in previously operated patients depends on the kind of prior surgery. The biopsy techniques do not represent a contraindication, whereas SLNB should not be applied after an extensive breast surgery neither in the context of oncologic nor non-oncologic purpose. It should neither be used after prior axillary surgery.

The adapted tumor size for the accurate SLNB should be restricted to $\mathrm{T} 1$ and $\mathrm{T} 2$. Within the limits of clinical trial this technique can be applicable in larger tumors like T3 and T4 and in the setting of preoperative chemotherapy.

No grants were given to support this paper.

\section{References}

1. Mansel RE, Fallowfield L, Kissin M, et al. Randomized multicenter trial of sentinel node biopsy versus standard axillary treatment in operable breast cancer: the ALMANAC Trial. J Natl Cancer Inst 2006; 98: 599-609.

2. Lyman $\mathrm{GH}$, Giuliano $\mathrm{AE}$, Somerfield MR, et al. American Society of Clinical Oncology guideline recommendations for sentinel lymph node biopsy in early-stage breast cancer. J Clin Oncol 2005; 23: 7703-7720.

3. Gump FE. Multicentricity in early breast cancer. Semin Surg Oncol 1992; 8: 117-121.

4. Vlastos G, Rubio IT, Mirza NQ, et al. Impact of multicentricity on clinical outcome in patients with T1-2, N0-1, M0 breast cancer. Ann Surg Oncol 2000; 7: 581-587.

5. Veronesi U, Paganelli G, Viale G, et al. Sentinel lymph node biopsy and axillary dissection in breast cancer: results in a large series. J Natl Cancer Inst 1999; 91: 368-373.

6. Hsueh EC, Turner RR, Glass EC, et al. Sentinel node biopsy in breast cancer. J Am Coll Surg 1999; 189: 207-213.

7. Krag DN, Weaver DL, Alex JC, Fairbank JT. Surgical resection and radiolocalization of the sentinel lymph node in breast cancer using a gamma probe. Surg Oncol 1993; 2: 335-339.

8. Giuliano AE, Kirgan DM, Guenther JM, Morton DL. Lymphatic mapping and sentinel lymphadenectomy for breast cancer. Ann Surg 1994; 220: 391-398.

9. McMasters KM, Wong SL, Martin RC, et al. Dermal injection of radioactive colloid is superior to peritumoral injection for breast cancer sentinel lymph node biopsy: results of a multi-institutional study. Ann Surg 2001; 233: 676-687.

10. Martin RC, Derossis AM, Fey J, et al. Intradermal isotope injection is superior to intramammary in sentinel node biopsy for breast cancer. Surgery 2001; 130: 432-438.

11. Kern KA, Rosenberg RJ. Preoperative lymphoscintigraphy during lymphatic mapping for breast cancer: improved sentinel node imaging using subareolar injection of technetium $99 \mathrm{~m}$ sulfur colloid. J Am Coll Surg 2000; 191: 479-489.

12. Klimberg VS, Rubio IT, Henry R, et al. Subareolar versus peritumoral injection for location of the sentinel lymph node. Ann Surg 1999; 229: 860-864.

13. Kern KA. Concordance and validation study of sentinel lymph node biopsy for breast cancer using subareolar injection of blue dye and technetium $99 \mathrm{~m}$ sulfur colloid. J Am Coll Surg 2002; 195: 467-475.

14. Tuttle TM, Colbert M, Christensen R, et al. Subareolar injection of $99 \mathrm{mTc}$ facilitates sentinel lymph node identification. Ann Surg Oncol 2002; 9: 77-81.

15. Chao C, Wong SL, Woo C, et al. Reliable lymphatic drainage to axillary sentinel lymph nodes regardless of tumor location within the breast. Am J Surg 2001; 182: 307-311.

16. Borgstein PJ, Meijer S, Pijpers J, van Diest PJ. Functional lymphatic anatomy for sentinel node biopsy in breast cancer: echoes from the past and the periareolar blue method. Ann Surg 2000; 232: 81-89.

17. Kumar R, Jana S, Heiba SI, et al. Retrospective analysis of sentinel node localization in multifocal, multicentric, palpable, or nonpalpable breast cancer. J Nucl Med 2003; 44: 7-10.

18. Schrenk P, Wayand W. Sentinel-node biopsy in axillary lymph-node staging for patients with multicentric breast cancer. Lancet 2001; 357: 122.

19. Tousimis E, Van Zee KJ, Fey JV, et al. The accuracy of sentinel lymph node biopsy in multicentric and multifocal invasive breast cancers. J Am Coll Surg 2003; 197: 529-535.

20. Knauer M, Konstantiniuk P, Haid A, et al. Multicentric breast cancer: a new indication for sentinel node biopsy-a multi-institutional validation study. J Clin Oncol 2006; 24: 3374-3380.

21. Kuehn T, Bembenek A, Decker $T$, et al. A concept for the clinical implementation of sentinel lymph node biopsy in patients with breast carcinoma with special regard to quality assurance. Cancer 2005; 103: 451-461.

22. Schwartz GF, Giuliano AE, Veronesi U. Proceedings of the consensus conference on the role of sentinel lymph node biopsy in carcinoma of the breast April 19-22, 2001, Philadelphia, Pennsylvania. Hum Pathol 2002; 33: 579-589.

23. Haigh PI, Hansen NM, Qi K, Giuliano AE. Biopsy method and excision volume do not affect success rate of subsequent sentinel lymph node dissection in breast cancer. Ann Surg Oncol 2000; 7: 21-27.

24. Ohtake E, Asaga T, Inaba M. Sentinel lymphoscintigraphy in patients with breast cancer undergoing excisional biopsy. Ann Nucl Med 2005; 19: 671-675. 
25. Tafra L, Lannin DR, Swanson MS, et al. Multicenter trial of sentinel node biopsy for breast cancer using both technetium sulfur colloid and isosulfan blue dye. Ann Surg 2001; 233: 51-59.

26. Luini A, Galimberti V, Gatti G, et al. The sentinel node biopsy after previous breast surgery: preliminary results on 543 patients treated at the European Institute of Oncology. Breast Cancer Res Treat 2005; 89: 159-163.

27. McMasters KM, Tuttle TM, Carlson DJ, et al. Sentinel lymph node biopsy for breast cancer: a suitable alternative to routine axillary dissection in multi-institutional practice when optimal technique is used. $J$ Clin Oncol 2000; 18: 2560-2566.

28. Albertini JJ, Lyman GH, Cox C, et al. Lymphatic mapping and sentinel node biopsy in the patient with breast cancer. JAMA 1996; 276: 1818-1822.

29. Krag D, Weaver D, Ashikaga T, et al. The sentinel node in breast cancer - a multicenter validation study. $N$ Engl $J$ Med 1998; 339: 941-946.

30. Borgstein PJ, Pijpers R, Comans EF, et al. Sentinel lymph node biopsy in breast cancer: guidelines and pitfalls of lymphoscintigraphy and gamma probe detection. J Am Coll Surg 1998; 186: 275-283.

31. Feldman SM, Krag DN, McNally RK, et al. Limitation in gamma probe localization of the sentinel node in breast cancer patients with large excisional biopsy. J Am Coll Surg 1999; 188: 248-254.

32. Miner TJ, Shriver CD, Jaques DP, Maniscalco-Theberge ME, Krag DN. Sentinel lymph node biopsy for breast cancer: the role of previous biopsy on patient eligibility. Am Surg 1999; 65: 493-498.

33. Wong SL, Edwards MJ, Chao C, et al. The effect of prior breast biopsy method and concurrent definitive breast procedure on success and accuracy of sentinel lymph node biopsy. Ann Surg Oncol 2002; 9: 272-277.

34. Port ER, Fey J, Gemignani ML, et al. Reoperative sentinel lymph node biopsy: a new option for patients with primary or locally recurrent breast carcinoma. J Am Coll Surg 2002; 195: 167-172.

35. Intra M, Trifiro G, Viale G, et al. Second biopsy of axillary sentinel lymph node for reappearing breast cancer after previous sentinel lymph node biopsy. Ann Surg Oncol 2005; 12: 895-899.

36. Port ER, Garcia-Etienne CA, Park J, et al. Reoperative sentinel lymph node biopsy: a new frontier in the management of ipsilateral breast tumor recurrence. Ann Surg Oncol. 2007 Epub.

37. Veronesi U, Paganelli G, Viale G, et al. A randomized comparison of sentinel-node biopsy with routine axillary dissection in breast cancer. $N$ Engl $J$ Med 2003; 349: 546-553.
38. Kim T, Giuliano AE, Lyman GH. Lymphatic mapping and sentinel lymph node biopsy in early-stage breast carcinoma: a metaanalysis. Cancer 2006; 106: 4-16.

39. Cox CE, Bass SS, McCann CR, et al. Lymphatic mapping and sentinel lymph node biopsy in patients with breast cancer. Annu Rev Med 2000; 51: 525-542.

40. Fraile M, Rull M, Julian FJ, et al. Sentinel node biopsy as a practical alternative to axillary lymph node dissection in breast cancer patients: an approach to its validity. Ann Oncol 2000; 11: 701-705.

41. Jakub JW, Pendas S, Reintgen DS. Current status of sentinel lymph node mapping and biopsy: facts and controversies. Oncologist 2003; 8: 59-68.

42. Chung $\mathrm{MH}, \mathrm{Ye} \mathrm{W}$, Giuliano AE. Role for sentinel lymph node dissection in the management of large $(\geqslant 5 \mathrm{~cm}$ ) invasive breast cancer. Ann Surg Oncol 2001; 8: 688-692.

43. Wong SL, Chao C, Edwards MJ, et al. Accuracy of sentinel lymph node biopsy for patients with T2 and T3 breast cancers. Am Surg 2001; 67: 522-526.

44. Bedrosian I, Reynolds C, Mick R, et al. Accuracy of sentinel lymph node biopsy in patients with large primary breast tumors. Cancer 2000; 88: 2540-2545.

45. Schwartz GF, Giuliano AE, Veronesi U. Proceedings of the consensus conference on the role of sentinel lymph node biopsy in carcinoma of the breast, April 19-22, 2001, Philadelphia, Pennsylvania. Cancer 2002; 94: 2542-2551.

46. Mamounas EP, Brown A, Anderson S, et al. Sentinel node biopsy after neoadjuvant chemotherapy in breast cancer: results from National Surgical Adjuvant Breast and Bowel Project Protocol B-27. J Clin Oncol 2005; 23: 2694-2702.

47. Stearns V, Ewing CA, Slack R, Penannen MF, et al. Sentinel lymphadenectomy after neoadjuvant chemotherapy for breast cancer may reliably represent the axilla except for inflammatory breast cancer. Ann Surg Oncol 2002; 9: 235-242.

48. Breslin TM, Cohen L, Sahin A, et al. Sentinel lymph node biopsy is accurate after neoadjuvant chemotherapy for breast cancer. J Clin Oncol 2000; 18: 3480-3486.

49. Kaufmann $M$, von Minckwitz $G$, Smith $R$, et al. International expert panel on the use of primary (preoperative) systemic treatment of operable breast cancer: review and recommendations. J Clin Oncol 2003; 21: 2600-2608.

50. Kaufmann M, Hortobagyi GN, Goldhirsch A, et al. Recommendations from an international expert panel on the use of neoadjuvant (primary) systemic treatment of operable breast cancer: an update. J Clin Oncol 2006; 24: 1940-1949. 